Southern Illinois University Edwardsville

SPARK

SIUE Faculty Research, Scholarship, and Creative Activity

$10-2016$

\title{
Content Blocking and the Patron as Situated Knower: What Would It Take for an Internet Filter to Work?
}

\author{
Richard Fry \\ Southern Illinois University Edwardsville \\ Emily Lawrence \\ University of Illinois at Urbana-Champaign
}

Follow this and additional works at: https://spark.siue.edu/siue_fac

Part of the Library and Information Science Commons, and the Other Philosophy Commons

\section{Recommended Citation}

Fry, Richard and Lawrence, Emily, "Content Blocking and the Patron as Situated Knower: What Would It Take for an Internet Filter to Work?" (2016). SIUE Faculty Research, Scholarship, and Creative Activity. 125.

https://spark.siue.edu/siue_fac/125

This Article is brought to you for free and open access by SPARK. It has been accepted for inclusion in SIUE Faculty Research, Scholarship, and Creative Activity by an authorized administrator of SPARK. For more information, please contact magrase@siue.edu. 


\title{
Content Blocking and the Patron as Situated Knower: What Would It Take for an Internet Filter to Work?
}

\author{
Emily Lawrence and Richard J. Fry
}

ABSTRACT

Librarians often object to Internet filters on the grounds that filters are prone to overblocking and underblocking. This argument implies that a significant problem with contemporary filters is that they are insufficiently fine-grained. In this article, we posit that present-day filters will always be conceptually capable of failure, regardless of how granular their content analysis becomes. This is because, we argue, objections to content are best understood as objections to problematic interactions between content and particular knowers. We import the concept of the situated knower from feminist epistemology to capture the heterogeneous, socially embedded nature of patrons, about whom we cannot make blunt generalizations for filtering purposes. A successful filter would need information about these differently situated patrons, the content they seek, and the interactions between the two. We conclude that a genuinely successful Internet filter would therefore need to be both mind reading and fortune-telling.

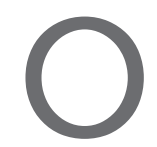

ne common objection to Internet filters is that they sometimes block content they should not block and fail to block content that they should block. These phenomena are known as overblocking and underblocking, respectively. The criticism that filters regularly overblock and underblock addresses both particular filtering policies and, more generally, manufacturer settings; it also presupposes that a sufficiently fine-grained filter that blocks by rote in accordance with an existing policy would be a successful filter. But is this the proper goal for an Internet filter? To put it another way: Even if existing Internet filters could be made to perfectly do what they are designed to do, would this constitute successful Internet filtering or merely successful blocking of particular (kinds of) content?

In this article, we articulate a conceptual answer to this question in the context of the library. Our answer stands in contrast to more common technical or policy-oriented ones. We argue that in order to genuinely succeed, given librarians' other goals and values, an Internet filter would have to take account of the role that the information sought would play in the

Library Quarterly: Information, Community, Policy, vol. 86, no. 4, pp. 403-418. (c) 2016 by The University of Chicago. All rights reserved. $0024-2519 / 2016 / 8604-0003 \$ 10.00$ 
seeker's life and purposes. This would require the ability to read patrons' minds and tell the future.

We argue that present-day, rote-blocking Internet filters are always conceptually capable of failure because there is no in-principle distinction between content that is objectionable and content that is unobjectionable in the absence of someone (in this case, the library patron) interacting with that content. Being objectionable, then, is a property of problematic relationships between content and knower, not a property of content in a vacuum. Here we introduce the situated knower, a concept from feminist social epistemology and standpoint theory, to capture the heterogeneous nature of knowers and the ways in which their social locations bear on what they (come to) know. We argue that thinking of patrons as situated knowers tracks librarians' thinking about the communities libraries serve. Patrons appear in the library as resolutely individual; we cannot make generalizations about content objectionability that will hold true over a whole population of knowers (or even parts of it). Contemporary, rote-blocking Internet filters can at best only implement rules based on generalizations about people and content. This means that it will always be possible for these filters to block content that they should not block (i.e., overblock), and fail to block content that they should block (i.e., underblock). We conclude that an ideal filter- that is, one that eliminates the possibility of over- or underblocking - would need to possess mind-reading and fortune-telling powers.

\section{The Trouble with (Contemporary) Internet Filters}

Internet filtering software is designed to restrict users' access to web content. There are varied methods for blocking content to meet this end. For instance, filtering software might limit access on the basis of keywords or text strings, scanned pixels, third-party site ratings, or some other information source (ALA 2000; DeCandido 2000).

Manufacturers began developing filtering software in the mid-19gos (Ayre 2004), largely to address growing anxiety about minors' perceived vulnerability to controversial and graphic content on the web. These concerns spurred legislation designed to protect children from purportedly harmful content. However, most of the censorship laws proposed in the late $1990 \mathrm{~s}$ were ruled unconstitutional; as a result, "parents, employers, school districts, and other government entities turned to privately manufactured Internet filters” (Heins, Cho, and Feldman 2006, 1).

The Children's Internet Protection Act (CIPA) emerged against this backdrop. CIPA was passed by Congress in 2000 and went into effect the following year (Menuey 2009). ${ }^{1}$ CIPA stipulates that public schools and libraries that receive discounts or grants via the Universal

1. The American Library Association famously opposed CIPA; however, in 2003 the Supreme Court ruled in United States vs. American Library Association that CIPA was not a breach of patrons' First Amendment rights (Menuey 2009). 
Service Fund for networked Internet access must block access to images that are obscene or "harmful to minors" (Dresang 2006, 180). For public libraries, this means filtering software must be installed on public computers if the library receives any of the following: "(1) E-rate discounts for Internet access costs, (2) E-rate discounts for internal connections costs, (3) Library Services and Technology Act (LSTA) funding for direct Internet costs, or (4) LSTA funding for purchasing technology to access the Internet" (Jaeger and McClure 2004).

CIPA sets out implicit criteria for filtering software, and librarians must choose from what is commercially available. Moreover, while CIPA's domain is exclusively adults' access to obscene content and minors' access to images that would harm them, overapplications of the law are common. It is frequently the case that, in implementing CIPA, institutions and individuals misunderstand it and the related case law (Caldwell-Stone 2013). Some believe that an institution must, to the greatest degree possible, block all potentially offensive sites. Others do not understand the conditions under which unblocking content or disabling filters for users is acceptable or "do not limit filtering to visual images as the law mandates" (Batch 2014, 11). Further, some fail to recognize the First Amendment ramifications of overapplications of CIPA (Caldwell-Stone 2013).

These overapplications notwithstanding, many librarians hold that filtering Internet access in public and school libraries runs directly counter to the stated ethical principles of librarianship and the profession's core value of intellectual freedom. The American Library Association (ALA) opposes Internet filters generally but also stands against any and all access restrictions based on a patron's age. According to one of ALA's interpretations of the Library Bill of Rights, "Free Access to Libraries by Minors": "Article V of the Library Bill of Rights states, 'A person's right to use a library should not be denied or abridged because of origin, age, background, or views.' The 'right to use a library' includes free access to, and unrestricted use of, all the services, materials, and facilities the library has to offer. Every restriction on access to, and use of, library resources, based solely on the chronological age, educational level, literacy skills, or legal emancipation of users violates Article V" (ALA 2010, 136).

The principles of librarianship generate a number of reasons to oppose filtering, and the practical problems with implementation further complicate matters. Significantly, Internet filters have been shown to overblock and underblock to varying degrees. In the early days of filtering (when simple keyword blocking was most prevalent), overblocking in particular was so common and the mechanisms by which software filtered content so unsophisticated that access to inarguably benign content was frequently restricted (e.g., Middlesex college and Dykes medical library [Heins et al. 2006, ii]).

In the intervening years, filtering software has become more context-sensitive (Ang 2007, 480). However, evidence suggests that filters continue to block content that they should not block with some regularity. In its 2006 policy report on Internet filters, the Brennan Center for Justice at New York University reviewed a number of large and small studies on filter effec- 
tiveness. On the whole, these studies found that even though filtering technology had improved, filters were "still seriously flawed" and continued to "deprive their users of many thousands of valuable web pages on subjects ranging from war and genocide to safer sex and public health" (Heins et al. 2006, ii).

University of California, Berkeley statistician Philip B. Stark performed a large-scale study of Internet filter effectiveness. This study (Stark 2008) was commissioned by the US Department of Justice in its defense of the Child Online Protection Act (COPA), a precursor to CIPA that never took effect. COPA would have restricted transmission of commercial web content thought to be "harmful to minors" (Lavell 2004). Stark examined a range of different blocking programs, testing how well each blocked "webpages with sexually explicit content." He found that "if the filter most effective at blocking adult materials were applied to search indexes, typical query results, or the results of popular queries, the number of clean pages blocked in error would exceed the number of adult pages blocked correctly" (Stark 2008, 411).

In a 2010 metastudy, librarian Sara Houghton-Jan looked at filtering studies from 2001-8 and determined that, with respect to blocking "objectionable content," filters had an average accuracy rate of about $78 \%$. Although that accuracy rate has risen in more recent years - it is $83 \%$ if you look exclusively at studies from 2007-8 - it is clear from Houghton-Jan's research that filters are in fact continuing to overblock and underblock content. Some filtering opponents also rightly call attention to specifically partisan values embedded in some filtering technology, a point evinced by filters that block certain sorts of "controversial" intellectual content, such as web pages on climate change or feminism. Others note that certain kinds of legitimate content are disproportionately vulnerable to overblocking, such as materials related to sexual health and lesbian, gay, bisexual, transsexual, or queer (LGBTO) resources (Greenblatt 2003; Heins et al. 2006; Alexander and Miselis 2007; Houghton-Jan 2010). ${ }^{2}$

Generally, practitioners who oppose filtering frame this as a failing of the technology itself, which they take to be insufficiently precise and overly susceptible to corporate manipulation (Ayres 2004; Houghton-Jan 2010). On this view, persistent limitations of filtering technology (and, sometimes, partisan values embedded in that technology, themselves perceived as a manifestation of technical limitations) result in overblocking and underblocking of content. Crucially, the notion that filters can in fact overblock or underblock entails a distinction between objectionable and unobjectionable content that is often left implicit. To understand

2. We focus on the practitioners' discourse surrounding Internet filters, which tends to emphasize over- and underblocking as central problems with filtering technology. However, scholars in information studies, media studies, science and technology studies, etc., have written extensively on the various ways in which digital technologies (necessarily) function as value-laden artifacts of power (see, for example, Pacey 1983; Sclove 1995; Friedman and Nissenbaum 1996; Fleischmann 2007; Brock 2011; Noble 2013). While Internet filters are apt for an analogous critique (especially in the library context), that is not the purpose of our work here. Rather, we seek to make a conceptual argument about the impossibility of filtering in accordance with any set of values. 
why contemporary filters are always conceptually capable of failure, we must first make that distinction explicit, clarifying what it means for content to be objectionable.

\section{What Makes Content Objectionable?}

People object to a range of different kinds of content for a range of different reasons, and accusations of objectionability form the basis for proposed restrictions to access. Some individuals, for instance, object to:

1. Materials about bomb building because they believe these materials facilitate amateur bomb construction.

2. Young adult (YA) literature with LGBTQ themes because they believe that this literature endorses harmful lifestyle choices.

3. Pornography because they believe it promotes violence against women.

Although the content is different in these three cases, they are all structurally similar. In each case someone raises an objection to some person accessing the content because access to that content by that person will—with some perceived probability — lead to some outcome thought to be bad by the objector. Usually the objection is raised by someone with a relevant interest in preventing that bad outcome.

These sorts of objections are often intelligible because we understand them as claims about the ways in which individuals interact with and take up content. As Emily Knox (2014) notes, "what knowledge one thinks should be accessible is intimately tied to one's assumptions of how that knowledge will affect the seeker" (11). Objections to content are linked to an individual's or group's beliefs about knowledge effects for particular seekers. Thus, in the three cases outlined above, we can see the objections as objections to:

1. Materials about bomb building when accessed by individuals inclined to build bombs that they will use to hurt innocent people (likely in their own community).

2. YA literature with LGBTQ themes when accessed by malleable children and adolescents whose moral education is still in progress.

3. Pornography when accessed by men and boys, perhaps specifically those with violent tendencies.

In case (1), where someone wants to restrict access to materials for individuals who are inclined to build bombs, it is unlikely that they retain their concern about the content in the case of, for example, a federal investigator who is evaluating publicly available web content related to bomb building. Similarly, the objector in case (2) who fears the effects on children and adolescents may not be concerned about access for queer and/or transgender adults. 
Ultimately, content in a vacuum is not what people typically object to, and this is because content is, in itself, not the right kind of thing to be objectionable or unobjectionable. It is not clear that we can have much at all to say about content without invoking a knowing subject. ${ }^{3}$ We will consider an objection to the claim we have just made before turning to the individuals who take in the content.

\section{An Objection to Interactions (and Not Content) as Problematic}

One might object to our claim that content is only objectionable relative to some subject by claiming that some content is so universally morally corrosive that it produces problematic interactions with every individual with whom it comes into contact. If there were to be such content, then that content would be objectionable simpliciter, this line of thinking goes. Two things are worth noting about this objection. First, the standard of evidence for showing that any such content actually exists will be quite high. Second, this objection grants the underlying premise of the argument rather than undermining it.

To take an example, one type of content that some judge to fit this bill is pornography. Challenges to pornographic material sometimes take it that the content is harmful to such an extent as to be unsafe for any individual to access for any purpose. Some think pornography is the social equivalent of nuclear waste: there is no use for it; it must be contained and never accessed for any reason. But establishing that pornography is dangerous is different from establishing the universal claim that it always produces problematic interactions. Several feminist cases against pornography provide exemplars of such arguments.

We take it that these are the contours of one standard feminist view: to successfully be pornography, the material must portray some or all of its subjects as mere objects of sexual exploitation, typically in a way that endorses that objectification. ${ }^{4}$ Viewing pornography induces participation in and propagation of this objectification, which results in concrete harm to women, and for this reason pornography ought to be avoided. Others believe that pornography causes men to engage in acts of violence and discrimination against women because they have (at least implicitly) come to see women as subordinate to them (MacKinnon 1987). These arguments straightforwardly do not meet the standard outlined above: they do not establish that any viewing of pornography produces problematic interactions, only that some might.

A more nuanced approach — taken by A. W. Eaton (2007) — takes a broader view of the class "pornography" and suggests that specifically inegalitarian pornography has a probabilistic causal relationship to a variety of individual and group harms that perpetuate gender inequality. ${ }^{5}$ But again, because this argument is probabilistic, it rests on the strength of the

3. It may indeed be the case that content cannot be content at all in the absence of some knower.

4. This is a view articulated by, e.g., Longino (1980).

5. "Just as we conceive of smoking's harms in probabilistic terms, so the hypothesis that pornography causes harm holds that men's exposure to pornography significantly increases the risk of a variety of harms to women" (Eaton 2007, 697). 
probability claims. If there were a way to know which individuals would perpetuate these harms after accessing pornography and which would not, then, in principle, the argument would not cut against pornography being accessed by individuals with whom it would not produce problematic interactions.

This is to say that it is not immediately clear whether a strong argument can be made that pornography has negative effects on all knowing subjects. One can make the case that pornography's harm has a high probability of resulting from viewing (and perhaps that that probability varies to some extent with respect to a viewer's cultural context), but that is not enough to guarantee that these effects are universal. ${ }^{6}$ Further, the causal relationships between pornography and, for example, violence against women are uncertain - that is, it is not clear whether viewing pornography causes the violence, or whether viewing pornography and committing violence are, for instance, effects of a common cause. These approaches do not make a strong enough case to conclude that the material in question is universally morally corrosive.

A different approach eschews discussions of harm altogether. Feminist philosopher Rae Langton (1990) centers her criticism of pornography on the idea that pornography conflicts with a consistent notion of democratic citizenry. Langton's approach has the beginnings of an argument for universal moral corrosiveness: Simply by taking in these representations of inegalitarian and objectifying relations, we compromise our ability to engage productively with others in a democratic, egalitarian society. Thus, pornography always produces an objectionable interaction - to every viewer under every circumstance-so we could consider it objectionable simpliciter. ${ }^{7}$

Langton's view brings us to the second point, though. It is important to see that objecting to some content because it always produces problematic interactions underlines our point about how content comes to be objectionable. The objectors in this case object to pornography precisely because they take it to produce problematic interactions; they just take those problematic interactions to be universally produced. This shows that calling some content objectionable simpliciter is really just shorthand for saying that it always produces problematic interactions. Thus, our claim that what objectors care about is problematic interactionsand not content per se-is vindicated.

So, as we continue on, we will suppose that when individuals object to content, they are objecting to interactions they foresee occurring between that content and some group of individuals. After we have addressed what we consider the typical class of objection-where the objector takes the content to produce problematic interactions only in some individuals

6. This is not to say that such arguments are not strong enough to count in favor of other sorts of moral interventions, but rather that restricting access to some class of content for all library patrons requires an argument applicable to all.

7. This returns us, though, to the question of what it means for some expression to be (a certain sort of) content and whether and to what extent this depends on the psychology of the particular viewer on a particular occasion. 
who access that content - we will return to this question of (purportedly) universally morally corrosive content.

\section{The Library Patron as Situated Knower}

We have argued that it is the way in which a particular individual interacts with some content, rather than the content in and of itself, that can be objectionable. It is the way the viewer's social, knowledge, motivational, and emotional states interact with the content that can underwrite objections to providing that content to that individual. The interactions between content and particular individuals are complex, and the situated knower, a core concept from feminist social epistemology, can help us understand that complexity.

A situated knower is "situated in particular relations to what is known and to other knowers"; this means that "what is known, and the way that it is known . . reflects the situation or perspective of the knower" (Anderson 2012). Feminist epistemologists developed the idea of the situated knower as a critical response to the anonymous, identical, and largely passive knowers of classical epistemology. Unlike these agents, a situated knower is embedded and positioned in ways that are ineluctably social in character. Gaile Pohlhaus (2012) describes the knower's situatedness as referring "to the situations in which the knower finds herself repeatedly over time due to the social relations that position her in the world" (717). Situatedness, then, captures the ways in which the knower is relationally located in a complex web of social patterns. ${ }^{8}$

Something like the concept of the situated knower is necessary to understanding library patrons as individuals and community members. Applying the concept to patrons allows us to more easily see them as differentiated knowers with diverse perspectives, (intersecting) identities, and divergent epistemic resources. Moreover, it reminds us that "individuals from different social locations have, to some extent, different experiences" and that "the ways that social location shapes experience are not homogenous within a particular social group" (Intemann 2010, 785). Patrons' social locations comprise multifarious social relations, and so their situatedness, in practice, often appears as a kind of resolute individuality to the librarian.

Whether or not librarians make use of the term of art "situated knower," many implicitly acknowledge their patrons as embedded in larger epistemic communities. It is rare that a good librarian will, upon reflection, refer to library users as generic or interchangeable, both because of the politics and ethics of librarianship and because the practical experiences librarians have with patrons often conflict with that perspective.

8. Feminist epistemologists have used the idea of the situated knower to examine in particular how gender qua social construct and other axes of oppression shape what we know and how we come to know it (Alcoff and Potter 1993). Many of these thinkers are also involved in a normative project in which they work to determine what role social factors ought to play in knowledge production. Those particular projects and their fruits are not relevant to our discussion here. 
Additionally, while the library and information science (LIS) literature does not often reference the "situated knower," the more fundamental concept of social epistemology arose within LIS. Margaret Egan coined the term "social epistemology," which first appears in print in the essay "Foundations of a Theory of Bibliography" (Egan and Shera 1952). Further, Egan and Jesse Shera "proposed social epistemology as a theoretical framework for the study of the production, distribution, and utilization of intellectual products" and in so doing articulated substantive epistemological commitments for LIS that are still relevant to library research and education today (Furner 2004, 793). ${ }^{9}$ As a result, the notion that knowledge is itself a phenomenon with significant social elements that demand investigation likely seems intuitive to many librarians and LIS scholars.

The concept of the situated knower is often associated with feminist standpoint theory, which centers on how marginalized perspectives - particularly those inhabited by womengenerate epistemic privilege when imbued with a kind of collective political consciousness. Standpoint theorists take it that knowledge is itself situated, and Donna Haraway (1988) famously argued as much. In opposition to the scientist's "god-trick," or claims to disembodied objectivity, Haraway articulated a theory of knowledge as necessarily partial and perspectival. Sandra Harding (2004), a foundational figure in feminist standpoint theory, builds on this idea when she explains that "to the extent that an oppressed group's situation is different from that of the dominant group, its dominated situation enables the production of distinctive kinds of knowledge" (7).

Standpoint theorists move beyond this claim about situatedness to make a yet stronger one: that these "distinctive kinds of knowledge" can have a special epistemic authority. Standpoint feminists posit that women's social position in relation to the dominant culture more readily allows them to develop a feminist standpoint, which in turn enables them to understand and critique patriarchal power relations, ideologies, and institutions (Hartsock 1983). Standpoint feminists have also taken on intersectional considerations, as when another originator of standpoint theory, Patricia Hill Collins (1986), explicates the value of a Black feminist standpoint, predicated partly on Black women's "outsider within status."

Standpoint feminists thus attribute epistemic advantage to specific marginalized standpoints. Contemporary standpoint theorists adopt a contextualist approach wherein different standpoints are advantageous under particular conditions and for particular purposes (Intemann 2010). Significantly, these standpoints do not result from social location alone. Cultivating a standpoint requires "critical conscious reflection on the ways in which power structures and resulting social locations influence knowledge production" (Intemann 2010, 785).

\footnotetext{
9. The notion that social epistemology could serve as a foundation for LIS is more contentious. Luciano Floridi (2002) critiques this position, arguing that philosophy of information is the foundation of LIS. Floridi does, however, acknowledge a robust relationship between LIS and social epistemology, which he takes it are in fact "siblings" whose "closeness . . . is better understood if explained in terms of a common origin, as two branches of [the philosophy of information], rather than hierarchically" (41).
} 
As Kathi Weeks (1998) explains, "a standpoint is a project, not an inheritance; it is achieved, not given" (136). One occupies a feminist standpoint, for instance, not merely by virtue of being a woman but through engagement in a shared, interpretive political project.

Given that we have described library patrons as situated knowers and that this particular concept is commonly associated with feminist standpoint theory, one might expect that we will go on to criticize Internet filtering technologies as products of cultural institutions that control the flow of information to reinforce existing structures of social power. That is, one might expect us to advance a standpoint feminist critique of filters, to explain how Internet filters are not politically neutral tools that can be implemented without appeal to hegemonic cultural norms, valued ways of knowing, and so on.

Internet filtering technologies are ripe for such an analysis, and while this would indeed be a worthwhile project, it is not our project here. Instead, we wish to make a critique that does not rely on the details of standpoint theory per se. We want to apply only the insight that, as Alison Wylie (2003) puts it, "social location systematically shapes and limits what we know, including tacit, experiential knowledge as well as explicit understanding" (31), and differently situated patrons will thus interpret and react in varied ways to information sources. That social location influences what we know is one of the core tenets of standpoint theory, but it is also available to theorists who do not attribute epistemic privilege to certain (usually marginalized) standpoints.

In short, our argument requires only this weaker claim that patrons are socially situated and that different social positions generate distinctive knowledge (not the stronger one regarding the development of epistemically advantaged standpoints). We think all LIS practitioners would agree to the basic spirit of this point, regardless of their views on any particular feminist epistemology. We believe this, in part, because references to the social nature of knowledge are found in works across LIS and are essential to, for example, reader response theory as well. ${ }^{10}$

While there are indeed trenchant criticisms that could be leveled from within a structurally complex version of feminist standpoint theory, explicating these criticisms is not the objective of this paper. Instead, we argue that the basic fact that patrons respond differently to the same sources leads to fundamental problems with rote-blocking Internet filters.

\section{Rote Filters Always Capable of Failure}

If objections to content are intelligible only in relation to situated knowers, this breeds problems that may be insurmountable for contemporary Internet filters that block by rote. An Internet filter's job is to prevent problematic interactions and to do so with a minimum of

10. See Knox (2014) for a discussion of contemporary librarianship's endorsement of the view that one cannot predict the effects of new knowledge. 
false positives. To do this, the filter would have to anticipate the ways in which particular patrons would interact with particular bits of content. The need for an Internet filter to know about patrons and the ways in which particular patrons would interact with the requested content presents unresolvable problems for filters that block by rote; filters that block according to a simple list of banned sites, keywords, or pixel colors are not sensitive to features of the patron's interaction with the content that would make that interaction objectionable or unobjectionable. Rote filters do not make judgments, and therefore it will always be possible for them to overblock and underblock. A rote filter has some access to information about the content itself but not to any information about the patron or how that patron is socially situated. Thus, rote filters will always be conceptually capable of failure: they are not suited to preventing problematic interactions while still maintaining a minimum of false positives.

Without detailed information about the patron and the ability to make judgments about qualities of the ensuing interaction between patron and content, it will always be possible for even the most finely tuned rote-blocking Internet filter to fail (i.e., to block content it ought not block or let through content that it should not). This is because, as it stands, filters block content based on blunt generalizations about users and about content.

\section{A Mind-Reading, Fortune-Telling Filter}

A filter that restricted access only in cases where not doing so would produce a problematic interaction would need to be able to assess the kind of interaction a particular patron would have with the particular content that patron requested. To do this, the filter would require accurate information about (1) the individual patron/knower, (2) the content itself, and (3) how these two would interact. This information is simply unavailable to contemporary Internet filters.

With regard to the individual patron/knower, a filter would need access to information about the patron's knowledge state, emotional state, intentions, interpretative powers, and social support system in order to judge what sorts of content would produce a problematic interaction with that patron. This is only a partial list, and yet it already presents myriad ethical, technical, and theoretical problems. Even if patrons willingly supplied much more and much more specific information about themselves than they currently do, a proper filter would still need access to information that patrons could not themselves supply: information about their (in some cases fluctuating and often introspectively unavailable) internal states and intentions, psychological background, and social support system; news and goings-on in their political community; and so on. In the end, the filter would need extensive information about the various forces and factors that constitute that patron's situatedness, even when these were epistemically inaccessible to the patron herself.

In addition, an ideal Internet filter — one that never overblocks or underblocks - would need a sophisticated understanding of the content it is asked to filter. Such information would 
go well beyond primary, secondary, or even tertiary categorization; the filter would need whatever information is required to determine what effects this particular item will have on some patron. It would need to understand the text itself (and its component parts), its subtext, and, further, any misreadings, incomplete readings, or uncharitable readings a patron might deploy. This is to say that a filter would need to know far more about a text and its features than it currently does, and that knowledge would go beyond the text's intended meaning or typical interpretation and how those often influence readers. This influence is not captured by simple categorization and requires specific attention to how different sorts of readers interpret and take up different works.

A filter would need to know about how texts influence patrons, generally, but it would also need to accurately predict how particular texts influence particular patrons. That is, in order to avoid overblocking and underblocking, a filter would need to be able to assess what kinds of consequences there would be for an individual — and the larger community — of this particular patron accessing this particular information at this particular time (and whether those effects are positive or negative as well as in compliance with its mission). A filter would need complex heuristics to govern its expectations about what kind of interaction to expect from the combination of the particular requested content with those particular states of the particular patron.

The upshot is that the filter would need an astounding amount of information, a keen evaluative faculty, and superb psychological judgment. In essence, in order to always block appropriately, an Internet filter - one capable of accessing information inaccessible to the patron herself and of evaluating polymorphic chains of effects - would need to possess powers of mind reading and fortune-telling. ${ }^{11}$

\section{Universally Morally Corrosive Content Not a Problem for the Viewer}

We must now return to the question of content that is purported to be universally morally corrosive. It would seem that content that was found to produce problematic interactions with every possible viewer could be blocked by rote, thus eluding our argument that filters will always overblock or underblock in the absence of mind reading, at least with respect to this particular content. There are three things to say about such an objection.

First, this argument for rote filtering from universal moral corrosiveness underlines the appropriateness of mind reading as the standard to which filters must be held. To try and block this content from ever being accessed based on the belief that it produces problematic

11. We hope that librarians will agree that there are numerous ethical problems with the kind of Internet filter we have described. That being said, there may be some important senses in which mind-reading filters are already being implemented in the library. This could occur when, e.g., librarians engage in Google-inspired search-optimization projects, or perhaps when the librarian must assess a patron's request to unblock some content. We hope to investigate these potential cases of mind reading in the future. 
interactions is to, in essence, do the mind reading ahead of time. In this way, the argument shows again that what is objectionable are certain sorts of interactions, and knowing features about the humans that figure into those interactions is crucial. The features in question are just-purportedly-universal in this case.

Second, an argument would have to be very strong to show that the features of knowing agents that make their interactions with such content in each case problematic are in fact universal. One would need to convincingly argue that the class of material in question would be damaging to any human viewer. That is, one would need to supply a compelling and universally applicable argument that this type of content is in each case problematic with respect to any viewer (and, further, that it serves no or insufficient positive purpose relative to anyone's information state). Some might think that the Langton view, as outlined earlier, does in fact show this. But it is far from being the consensus, so more work would have to be done to establish this result.

Third, such an argument, if successful, would merely return us to the problems with contemporary rote-blocking filters. This is because, in an attempt to block that class of content entirely, we would in essence be blocking by rote. Overblocking and underblocking would thus, presumably, occur at the same problematic rate that they do today. Producing an argument that some class of content is universally morally corrosive does not solve the problems others have identified with rote-blocking filters and so does not license filtering, given current technical limitations and the unacceptability of overblocking.

\section{Conclusion}

A common objection to current Internet filtering technologies is that they frequently allow content through that should be blocked (according to the desires of those implementing the filtering policy) and frequently block content that is unobjectionable. Filters, as they stand, are treated as being insufficiently fine-grained in their discriminatory powers to do the task to which they are set, and this insufficiency is often the basis of arguments against them. However, we have argued here that a rote filter cannot in fact be sufficiently fine-grained to preclude overblocking and underblocking. Because patrons are all situated differently and what we seek to prevent are problematic interactions, filters need access to information about patrons, content, and the interactions between the two to do the job that they are supposed to do. Collecting this information is both (presently) impossible and fraught with moral complications. Our hope is that this article will pave the way for future discussions of these complications, particularly with reference to their import for present-day libraries and information policy. We also anticipate that our conclusions will have significant rhetorical and argumentative implications for librarians as they continue to make the worthwhile case against Internet filtering. 


\section{References}

Alcoff, Linda, and Elizabeth Potter, eds. 1993. Feminist Epistemologies. New York: Routledge.

Alexander, Linda B., and Sarah D. Miselis. 2007. "Barriers to GLBTQ Collection Development and Strategies for Overcoming Them." Young Adult Library Services 5 (3): 43-49.

ALA. 2000. "Statement on Library Use of Filtering Software." http://www.ala.org/advocacy/intfreedom /statementspols/statementlibrary.

ALA. 2010. Intellectual Freedom Manual. 8th ed. Chicago: American Library Association.

Anderson, Elizabeth. 2012. "Feminist Epistemology and Philosophy of Science." The Stanford Encyclopedia of Philosophy, edited by Edward N. Zalta. http://plato.stanford.edu/archives/fall2012/entries/feminism -epistemology.

Ang, Peng Hwa. 2007. "Censorship of the Internet." Encyclopedia of Library and Information Science. 2nd ed. New York: Taylor \& Francis.

Ayre, Lori Bowen. 2004. "Filtering and Filter Software." Library Technology Reports 40 (2): 5-80.

Batch, Kristen R. 2014. Fencing Out Knowledge: Impacts of the Children's Internet Protection Act 10 Years Later. Chicago: American Library Association.

Brock, André. 2011. "Beyond the Pale: The Blackbird Web Browser's Critical Reception." New Media and Society 13 (7): 1085-103.

Caldwell-Stone, Deborah. 2013. "Filtering and the First Amendment: When Is it Okay to Block Speech Online?" American Libraries (March/April). http://www.americanlibrariesmagazine.org/article/filtering -and-first-amendment.

Collins, Patricia Hill. 1986. "Learning from the Outsider Within: The Sociological Significance of Black Feminist Thought." Social Problems 33 (6): S14-S32.

DeCandido, GraceAnne A. 2000. "Filtering: No Easy Answers." Public Libraries 39 (2): 78-79.

Dresang, Eliza T. 2006. "Intellectual Freedom and Libraries: Complexity and Change in the Twenty-FirstCentury Digital Environment." Library Quarterly 76 (2): 169-92.

Eaton, A. W. 2007. "A Sensible Antiporn Feminism." Ethics 117:674-715.

Egan, Margaret, and Jesse Shera. 1952. "Foundations of a Theory of Bibliography." Library Quarterly 22 (2): $125-37$.

Fleischmann, Kenneth. 2007. "Digital Libraries with Embedded Values: Combining Insights from LIS and Science and Technology Studies." Library Quarterly 77 (4): 409-27.

Floridi, Luciano. 2002. "On Defining Library and Information Science as Applied Philosophy of Information." Social Epistemology 16 (1): 37-49.

Friedman, Batya, and Helen Nissenbaum. 1996. "Bias in Computer Systems.” ACM Transactions on Information Systems 14 (3): 330-47.

Furner, Jonathan. 2004. "'A Brilliant Mind': Margaret Egan and Social Epistemology." Library Trends 52 (4): 792-809.

Greenblatt, Ellen. 2003. "Lesbian, Gay, Bisexual, Transgender Library Users: Overcoming the Myths." Colorado Libraries 29 (4): 21.

Haraway, Donna. 1988. "Situated Knowledges: The Science Question in Feminism and the Privilege of Partial Perspective." Feminist Studies 14 (3): 575-99.

Harding, Sandra, ed. 2004. The Feminist Standpoint Theory Reader: Intellectual and Political Controversies. New York: Routledge. 
Hartsock, Nancy. 1983. "The Feminist Standpoint: Developing the Ground for a Specifically Feminist Historical Materialism." In Discovering Reality: Feminist Perspectives on Epistemology, Metaphysics, Methodology, and Philosophy of Science, edited by Sandra Harding and Merrill B. Hintikka. Dordrecht: Kluwer.

Heins, Marjorie, Christina Cho, and Ariel Feldman. 2006. Internet Filters: A Public Policy Report, Brennan Center for Justice. New York: Brennan Center for Justice at New York University School of Law.

Houghton-Jan, Sarah. 2010. "Why Internet Filters Don't Work and Why Libraries Who Filter Are Wrong." Librarian in Black. http://librarianinblack.net/librarianinblack/filtering/.

Intemann, Kristen. 2010. "25 Years of Feminist Empiricism and Standpoint Theory: Where Are We Now?" Hypatia 25 (4): 778-96.

Jaeger, Paul T., and Charles R. McClure. 2004. "Potential Legal Challenges to the Application of the Children's Internet Protection Act (CIPA) in Public Libraries: Strategies and Issues." First Monday 9 (2). http://firstmonday.org/ojs/index.php/fm/article/view/1117/1037.

Knox, Emily. 2014. "Supporting Intellectual Freedom: Symbolic Capital and Practical Philosophy in Librarianship." Library Quarterly 84 (1): 8-21.

Langton, Rae. 1990. "Whose Right? Ronald Dworkin, Women, and Pornographers." Philosophy and Public Affairs 19 (4): 311-59.

Lavell, Amy Lisewski. 2004. "In the Name of In(ternet)decency: Laws Attempting to Regulate Content Deemed Harmful to Children." Public Libraries 43 (6): 353-59.

Longino, Helen. 1980. "Pornography, Oppression, and Freedom: A Closer Look." Take Back the Night. New York: William Morrow.

MacKinnon, Catharine. 1987. Feminism Unmodified: Discourses on Life and Law. Cambridge, MA: Harvard University Press.

Menuey, Brendan P. 2009. "CIPA: A Brief History." Computers in Schools: Interdisciplinary Journal of Practice, Theory, and Applied Research 26 (1): 40-47.

Noble, Safiya. 2013. "Google Search: Hyper-visibility as a Means of Rendering Black Women and Girls Invisible." InVisible Culture: An Electronic Journal for Visual Culture 19. http://ivc.lib.rochester.edu/google -search-hyper-visibility-as-a-means-of-rendering-black-women-and-girls-invisible.

Pacey, Arnold. 1983. The Culture of Technology. Cambridge, MA: MIT Press.

Pohlhaus, Gaile. 2012. "Relational Knowing and Epistemic Injustice: Toward a Theory of Willful Hermeneutical Ignorance." Hypatia 27 (4): 715-35.

Sclove, Richard E. 1995. Democracy and Technology. New York: Guilford.

Stark, Philip B. 2008. "The Effectiveness of Internet Content Filters." I/S: A Journal of Law and Policy for the Information Society 4 (2): 411-30.

Weeks, Kathi. 1998. Constituting Feminist Subjects. Ithaca, NY: Cornell University Press.

Wylie, Alison. 2003. "Why Standpoint Matters." In Science and Other Cultures: Issues in Philosophies of Science and Technology, edited by Sandra Harding and Robert Figueroa. New York: Routledge.

Emily Lawrence: doctoral student, Graduate School of Library and Information Science, University of Illinois at Urbana-Champaign. Lawrence's research focuses on the ways in which aesthetics can inform ongoing ethical and conceptual disputes in LIS. Lawrence's dissertation defends an aesthetic 
education model for readers' advisory services. Lawrence holds an MLS with a specialization in information and diverse populations from the University of Maryland. Prior to beginning the doctoral program at Illinois, Lawrence worked in reference and web services at the National Library of Medicine. E-mail (corresponding author): elawrnc2@illinois.edu.

Richard J. Fry: assistant professor, Southern Illinois University, Edwardsville. Fry received his PhD in philosophy from Georgetown University. His research interests center on early modern philosophy (especially the philosophy of David Hume) and philosophy of science. Fry addresses issues surrounding knowledge, privacy, and artificial intelligence in his introductory philosophy courses. His most recent research looks at the debates surrounding the cognitive capabilities of nonhuman animals in early modern philosophy. E-mail: rfry@siue.edu. 